\title{
Clinical effectiveness - a series of perspectives
}

\author{
Sarah Marriott
}

Ensuring clinically effective practice and service models is now a major challenge for the National Health Service. It requires a clear focus on the purpose of healthcare provision - care which is most likely to result in the best possible individual or population outcomes within existing resources.

There are many people whose decisions affect whether or not care provided is effective: clinicians, policy-makers, commissioners, managers, patients and carers. If their decisions are to be influenced by a knowledge of what is likely to work best, they need ready access to the best available information and, in some instances, to develop their skills in interpreting it and using it appropriately. Methods for systematically identifying the highest quality evidence, bringing it to the attention of those who may require it, educating and training those who could use it, and supporting the changes necessary to implement it in routine practice have all become areas of research in their own right.

The success of clinical effectiveness will depend on better coordination of healthcare practices and their delivery, within teams, between departments and across organisations, as well as a willingness to collaborate between those taking decisions, be they clinicians, researchers, managers or patients. At the same time, however, we find ourselves working in an increasingly complex environment. While our approach to psychiatric practice has always been underpinned by the basic sciences and primary research, we also practise within ethical and legal frameworks, apply the benefit of our experience, and consider the patient's preference in deciding what care to provide in any individual case. In reality, the evidence base for practice is extensive, diverse and sometimes difficult to summarise.

Best clinical practice lies at the heart of clinically effective practice. Clinicians need to understand the emerging issues, and the surrounding controversies, if they are to remain central in determining the appropriateness of care provided to their patients. This series explores clinical effectiveness from a number of perspectives, and in so doing aims to contribute to this important debate.

Sarah Marriott, Research Fellow, Royal College of Psychiatrists, College Research Unit, 11 Grosvenor Crescent, London SWIX 8PG 